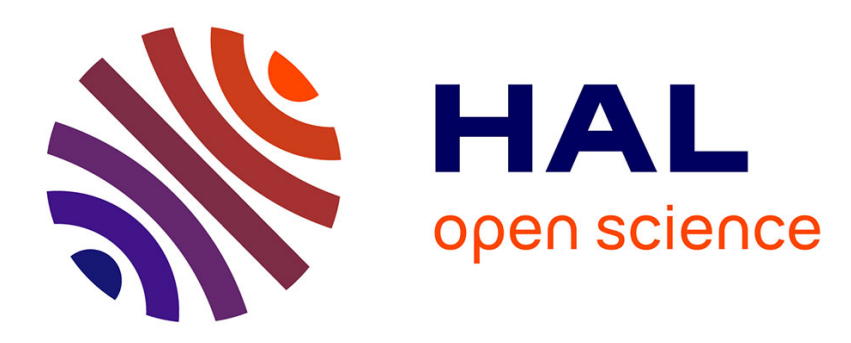

\title{
Analytical micromechanical models for the prediction of multiple cracking in composites
}

\author{
L. Mccartney
}

\section{To cite this version:}

L. Mccartney. Analytical micromechanical models for the prediction of multiple cracking in composites. Journal de Physique IV Proceedings, 1993, 03 (C7), pp.C7-1637-C7-1646. 10.1051/jp4:19937256 . jpa-00251895

\section{HAL Id: jpa-00251895 https://hal.science/jpa-00251895}

Submitted on 1 Jan 1993

HAL is a multi-disciplinary open access archive for the deposit and dissemination of scientific research documents, whether they are published or not. The documents may come from teaching and research institutions in France or abroad, or from public or private research centers.
L'archive ouverte pluridisciplinaire HAL, est destinée au dépôt et à la diffusion de documents scientifiques de niveau recherche, publiés ou non, émanant des établissements d'enseignement et de recherche français ou étrangers, des laboratoires publics ou privés. 


\title{
Analytical micromechanical models for the prediction of multiple cracking in composites
}

\author{
L.N. McCARTNEY \\ Division of Materials Metrology, National Physical Laboratory, Teddington, Middx. TW11 OLW, U.K.
}

\begin{abstract}
Because of the wide variety of composite materials that are available, and the expense involved in testing to measure properties for design purposes (such as their damage tolerance), there is a need to develop predictive methods that can readily be utilised on desk-top computers. It is inevitable that such methods will be based on analytical rather than finite element techniques. A review is given of recent progress that is being made with the use of analytical micromechanical models for the prediction of the dependence of thermoelastic constants on crack density, and for the prediction of multiple cracking in both unidirectional and laminated composites.
\end{abstract}

Various stress transfer models for unidirectional and laminated composites are assessed for their quality by considering their ability (or otherwise) to satisfy the equations of equilibrium, stress-strain relations, interfacial boundary conditions, and external boundary conditions. Both shear-lag and variational models are considered. In addition, so-called complete solution models are described that provide both the stress and displacement distributions in a composite. The characteristics of the various models are briefly described.

A most important need is to be able to predict the dependence of the thermoelastic constants on the level of applied stress or strain rather than on crack density so that non-linear stress-strain behaviour can be predicted. A recently developed energetic method of predicting multiple cracking in laminated composites is described showing how it can deal with both the simultaneous and progressive formation of cracks during loading for combined biaxial and shear modes of loading. It is pointed out that the fracture criterion can be derived from energetic principles or from the application of the generalised plane strain model of stress transfer, thus demonstrating energetic consistency. The fracture criterion depends only on macroscopic parameters, namely, the thermoelastic constants of cracked laminates and it follows that micromechanical models are only needed to determine the dependence of these constants on crack density. 


\section{INTRODUCTION}

Because of the large number parameters determining the properties of composites, and because of the significant expense involved in testing to measure properties, there is a very strong driving force to develop methods of predicting composite properties from the properties of their constiments. Unlike measurement methods, predictive techniques can quickly estimate the benefits that might accrue from the introduction of new fibres or matrices that are candidates for incorporation into composite materials and structures. Properties that are of interest are the elastic constants and thermal expansion coefficients of undamaged composites and of those containing some form of damage such as fibre fractures and matrix cracks in unidirectional composites, and transverse cracks in laminates. The prediction of damage initiation, e.g. first matrix cracking or ply cracking, is a very important requirement. This is followed by the prediction of damage growth as the applied load is increased.

There are two types of predictive technique that are commonly used in the technical literature. First of all, use has been made of numerical techniques such as finite element methods. Such techniques attempt to satisfy all the equations of elasticity theory and the boundary conditions that are necessary for the solution to be unique. For composite materials, especially those that are unidirectional containing some form of damage, it is very difficult to obtain solutions for arrays of fibres (e.g. either square or hexagonal packing) due to the excessive number of finite elements needed to represent the geometry. Representative volume elements have to be used which means that edge effects cannot easily be investigated. If composites having a range of volume fractions need to be analysed then new finite element meshes need to be created for each volume fraction: a time consuming activity. Finite element techniques usually lead to discontinuities of the stress field between each element of the system, and such discontinuities at perfectly bonded interfaces between fibres and matrix of unidirectional composites or between the plies of laminates, will be of concern. For example, the exact solution has singularities at such locations. Although finite element techniques offer the best prospects for estimating accurate solutions for any geometry, numerical solutions need to be carried out on mainframe computers or work-stations using sophisticated and expensive software packages that must be run by specially trained experts. There is a need to develop user-friendly predictive techniques that can be implemented on desk-top PCs, using the second type of predictive technique that is based on analytical models.

An analytical model for the prediction of the properties of composites is one that uses mathematical analysis (such as calculus and algebra) to solve the equations of elasticity, usually approximately. The results of calculations are mathematical formulae rather than tables of numerical results that are produced by the finite element method. The formulae can easily be programmed on a PC providing the user with a cost effective method of estimating composite properties. To develop an analytical model it is usually necessary to introduce approximations. There are a very large number of permutations that can be made of various approximations needed to develop analytical models. The technical literature abounds with analytical models based on a bewildering number of such approximations. The first objective of this paper is to review some of the analytical models that have been developed. The second objective is to show how high quality models of stress transfer can be used to develop relatively simple equations that govern the initiation and growth of cracks in composites subject to complex loading modes.

\section{STRESS TRANSFER MODELS}

When some form of damage is present in a unidirectional composite, stress is transferred between fibres and matrix in the neighbourhood of the damage such as fibre fractures or matrix cracks. For laminates, stress transfer occurs between neighbouring plies in the vicinity of transverse cracks. Damage leads to a degradation of properties that is controlled by the way in which stress redistribution occurs. It is therefore 
vital to be able to predict stress transfer phenomena accurately using models that can predict the local stress and displacement distributions in damaged composites. Analytical models that attempt to achieve this will now be described.

\section{Shear-lag models}

Shear-lag models, first introduced by Cox [1], have received the most attention in the technical literature. It is not possible to review here the many variants of shear-lag theory. It is more useful to indicate the best type of shear-lag approach, and to identify the approximations that have to be made. For low volume fraction composites, such as those used for fibre fragmentation or pull-out test, the original theory of Cox [1] should be used. For unidirectional composites (having volume fractions in the range $0.3 \leq V_{f} \leq 0.7$ ) that are perfectly bonded, Nayfeh [2] has developed the most efficient shear-lag model by employing an averaging procedure for fibre and matrix regions. For perfectly bonded laminates Nuismer and Tan [3] have used a similar averaging procedure to develop a corresponding shear-lag model. McCartney [4] has discussed the latter two shear-lag models in detail and identified the following approximations that need to be made:

\section{UD-model:}

* only one of the two equilibrium equations is retained and it is averaged over the cross-sections of fibre and matrix to generate two equations that determine the distribution of average axial stresses in fibre and matrix,

* the shear stress-strain relation is approximated by neglecting variations of radial displacement on the axial coordinate,

* only the axial stress-strain relation is retained and it is averaged over the cross-sections of fibre and matrix, neglecting the effect of transverse stress components.

\section{Laminate-model (0-90-0):}

* only one of the two equilibrium equations is retained and it is averaged over the cross-sections of the $0^{\circ}$ and $90^{\circ}$ plies to generate two equations that determine the distribution of average axial stresses in each ply,

* the shear stress-strain relation is approximated by neglecting variations of through-thickness displacement on the through-thickness coordinate,

* only the axial stress-strain relations is retained and it is averaged over the cross-sections of the $0^{\circ}$ and $90^{\circ}$ plies, neglecting the effect of transverse stress components.

Both the unidirectional and cross-ply laminate shear-lag models reduce the elasticity problem to solving a second order ordinary differential equation of the form:

$$
\tau^{\prime \prime}(\mathrm{z})=\mathrm{k}^{2} \tau,
$$

where $\tau$ is the interfacial shear stress, $\mathrm{z}$ is the axial coordinate, and where $\mathrm{k}$ is a material constant depending upon the properties and geometry of the composite constituents (see [4] for details). 


\section{Variational models}

There are three useful variational techniques that can be applied in elasticity theory. The first, known as the principle of minimisation of potential energy, is based on a representation for displacements (satisfying prescribed applied displacement conditions) which define stresses through the use of the stress-displacement relations. The variational principle asserts that potential energy will be a minimum only if the equations of equilibrium are satisfied. The second principle is the minimisation of complementary energy based upon a representation of the stresses (satisfying prescribed applied traction conditions) that satisfies the equilibrium equations. This variational principle asserts that complementary energy will be a minimum if the stress-displacement relations are satisfied. The third principle developed by Reissner [5] allows both stresses and displacement to be varied subject to boundary conditions involving both applied displacements and tractions. The Reissner energy function is a minimum only if both the equilibrium and stressdisplacement relations are satisfied.

Variational techniques have proved to be very useful when predicting the thermoelastic constants of both unidirectional and cross-ply composites. Variational models are most often based upon the concept of minimising complementary energy, the density function of which is expressed in terms of stresses. Hashin [6] used the technique to predict the stress distribution in 0-90-0 laminates containing a regular array of transverse cracks, and the dependence of the elastic constants on transverse crack density. Hashin [7, 8] later used the technique to predict the thermal expansion coefficients and to investigate orthogonal cracking.

For unidirectional composites Nairn [9] has recently developed a variational method that is entirely analogous to that used earlier by Hashin [7] for laminates. The characteristics of these variational models may be summarised as follows:

* a representation of the stress field is used that satisfies exactly the equilibrium equations and boundary and interface conditions involving stresses,

* the elastic constants are calculated by requiring that the effective energy density of a cracked laminate has the same form as that of an undamaged laminate,

* no prediction can be made of displacement field corresponding to the stress distribution derived by the technique,

* the elasticity problem is reduced to solving a fourth order ordinary differential equation of the form

$$
P C^{\prime \prime \prime \prime}(\mathrm{z})+Q C^{\prime \prime}(\mathrm{z})+\mathrm{RC}(\mathrm{z})=0 \text {, }
$$

where $C(z)$ is the function characterising the assumed stress distribution for the variational calculation and where the coefficients $P, Q$ and $R$ are material constants that depend upon the properties and geometry of the constituents of the composite.

\section{Complete solution techniques}

Neither shear-lag nor variational methods provide solutions that enable the prediction of the distributions of all stress and displacement components throughout the composite system. Shear-lag theory neglects relevant stress and displacement components while variational methods do not predict the displacement distribution. Techniques are needed to predict the complete solution and some of these will now be described briefly. Readers are, however, also referred to the work of Pagano [10,11,12] who has applied 
the Reissner variational principle to unidirectional and laminated composites solving for the stress field and the weighted average displacements.

\section{Unidirectional composites:}

\section{Model I:}

McCartney [13] has developed a complete but approximate solution for unidirectional composites. The solution technique developed has the following characteristics:

* the axial stresses in fibres and matrix are assumed to depend only on the axial coordinate.

* the equilibrium equations are satisfied exactly,

* the interface conditions for both stresses and displacements are satisfied exactly,

* the relevant shear stress-strain relation is satisfied exactly,

* the remaining relevant stress-strain relations ( 3 in number) are satisfied in an average sense by averaging over the cross-sections of fibre and matrix,

* the applied traction boundary conditions are satisfied exactly, and the applied displacement boundary conditions are satisfied in an average sense,

* the elasticity problem is reduced to solving a fourth order ordinary differential equation of the form

$$
\mathrm{P}^{\prime} \mathrm{C}^{\prime \prime \prime \prime}(\mathrm{z})+\mathrm{Q}^{\prime} \mathrm{C}^{\prime \prime}(\mathrm{z})+\mathrm{R}^{\prime} \mathrm{C}(\mathrm{z})=0,
$$

where $C(z)$ is the function characterising the stress and displacement distributions and where the coefficients $\mathrm{P}^{\prime}, \mathrm{Q}^{\prime}, \mathrm{R}^{\prime}$ are material constants that depend upon the properties and geometry of the constituents of the composite.

Model I has been established for the case where fibre and matrix are isotropic solids [13], and for the case when they are transverse isotropic [4].

\section{Model II:}

McCartney [14] has recently modified the earlier approach [13] used when developing model I by making use of Nairn's representation for the stress field [9] (used in a variational calculation), and then deriving the corresponding displacement field. The resulting complete solution is such that:

* the axial stresses in fibres and matrix are assumed to depend only on the axial coordinate; the only assumption of the model that leads to the following characteristics of the solution,

* equilibrium equations are satisfied exactly,

* interface conditions are satisfied exactly, 
* all stress-strain relations are satisfied exactly apart from the axial relation that is satisfied in an average sense (averaging across cross-section of the fibre, and of the matrix),

* all external traction boundary conditions are satisfied exactly, and displacement conditions are satisfied in an average sense,

* the problem reduces to solving a differential equation of the form (3),

* the solution minimises the Reissner energy function,

* model II is the best possible that can ever be developed based upon the single fundamental assumption that axial stresses depend only on the axial coordinate.

\section{Multiple cylinder model:}

The third stress transfer model that will be described is an extension of model $I[4,13]$ so that multiple concentric cylinders can be considered. There are two reasons for this extension. First of all, the model can deal with the effects of coatings, transcrystalline layers, or of reaction product layers that are applied or form at fibre/matrix interfaces. Secondly, composites can be analysed in much more detail by representing the fibre by a series of concentric cylinders, the matrix by another set of cylinders, and by representing surrounding composite material by yet another set of cylinders. The model can also be used to analyse fibre pull-out problems where a single fibre is embedded within a large sample of matrix. For such problems the fibre and matrix can be represented by a series of perfectly bonded concentric cylinders.

By increasing the number of cylinders representing the various phases present in the problem, solutions of increasing accuracy can be obtained. Errors introduced in the model by approximation will reduce in magnitude and solutions tend to the exact solution. To date a multiple cylinder model has been established only for a perfectly bonded composite [15]. Preliminary solutions show that the technique is very robust and can indicate the nature of the singularities that occur on the fibre/matrix interface at the location of either fibre fractures or matrix cracks. The multiple cylinder model has also been validated by comparison of predictions with corresponding finite element results [15].

\section{Cross-ply laminates:}

McCartney [16] has developed a generalised plane strain model of stress transfer for cross-ply laminates, and it has been shown that this complete solution technique leads to exactly the same differential equation (2) as the variational calculation when carried out for generalised plane strain conditions. Pagano [17] has shown that the approximations used, when deriving the complete solution for the generalised plane strain model of 0-90-0 laminates, are such that the Reissner energy function would be minimised when carrying out a variational calculation. The conclusion is that the generalised plane strain model of stress transfer is the best possible that can ever be developed based upon the single fundamental assumption that axial stresses depend only on the axial coordinate. The generalised plane strain model has the same characteristics as model II for unidirectional composites, where averages are now taken over the crosssections of the $0^{\circ}$ and $90^{\circ}$ plies, rather than over the fibre and matrix.

For in-plane shear deformation, Hashin [6] has provided the appropriate stress distribution that minimises the complementary energy. The corresponding displacement field can easily be found using a corresponding approach to that given in [16]. 


\section{PREDICTION OF CRACKING IN UNIDIRECTIONAL AND 0-90-0 CROSS-PLY LAMINATES}

The development of high quality complete solution models of stress transfer described above is leading to new analytical formulae that predict the onset and growth of cracking in both unidirectional and laminated cross-ply composites for complex loading situations involving both biaxial and shear loading. One case will be considered here, namely, transverse cracking in cross-ply laminates [18]. Consideration will be restricted to the case of perfect bonding. Applications of the methodology to matrix cracking in unidirectional composites, including with other interface conditions, such as the constant interfacial shear stress model and the Coulomb friction law, are in progress.

Following the principles established by Aveston, Cooper and Kelly [19], a general energy balance method is used which can be expressed for either unidirectional or laminated composites in the form:

$$
\Delta \Gamma+\Delta \mathrm{F}=\Delta \mathrm{W},
$$

where $\Delta \Gamma$ is the energy absorbed by the formation of new fracture surfaces, $\Delta F$ is the increase in Helmholz free energy of the system resulting from crack formation, and where $\Delta \mathrm{W}$ is the increment of work done by the applied tractions when the new cracks form. It should be noted that Helmholtz free energy has been used in place of strain energy as thermal expansion mismatch effects and the resulting residual stresses are to be taken into account by the model. If it is assumed that the composite is deformed under load control so that the applied tractions can be regarded as being held fixed during the formation of cracks, then the energy balance equation can be written in the following simpler form:

$$
\Delta \Gamma+\Delta G=0 \text {, }
$$

where $\Delta G$ is the increase in Gibbs free energy (a negative quantity) resulting from crack formation.

For a cross-ply laminate containing $\mathrm{n}$ cracks per unit length, assuming that the cracks traverse the entire cross section of the $90^{\circ}$ ply in cross-ply laminates, the Gibbs free energy per unit volume $\mathrm{g}$ may be expressed:

$$
g=-\frac{1}{2}\left[\frac{\left(\sigma-\sigma_{0}\right)^{2}}{E_{A}(n)}+\frac{\left(\sigma_{t}-\sigma_{o}^{*}\right)^{2}}{E_{T}(n)}-\frac{2 v_{A}(n)}{E_{A}(n)}\left(\sigma-\sigma_{o}\right)\left(\sigma_{t}-\sigma_{o}^{*}\right)+\frac{\tau^{2}}{\mu_{A}(n)}\right]+g_{0}(\Delta T),
$$

where $\sigma$ and $\sigma_{\mathrm{t}}$ are the effective axial and transverse applied stress respectively, and where $\tau$ is the applied shear stress. The stresses are effective in the sense that they are regarded as averages over the laminate edges corresponding to uniform applied displacements that would, in a homogeneous solid, lead to homogeneous axial strains $\varepsilon$, transverse strains $\varepsilon^{*}$ and shear strains $\gamma$. The terms $\sigma_{0}$ and $\sigma_{0}{ }^{*}$, that depend upon the difference $\Delta \mathrm{T}$ between the current temperature and the stress-free temperature, are thermal residual stresses arising from thermal expansion mismatch effects (see [18] for details). The function $g_{0}(\Delta T)$ is the value of $g$ when $\sigma=\sigma_{0}, \sigma_{1}=\sigma_{0}^{*}$ and $\tau=0$. The parameters $\sigma_{0}$ and $\sigma_{0}^{*}$ are in fact the values of the applied stresses $\sigma$ and $\sigma_{\mathrm{t}}$ respectively such that any cracks in the $0^{\circ}$ and $90^{\circ}$ plies are just closed. The parameters $E_{A}(n), E_{T}(n), \mu_{A}(n)$ and $v_{A}(n)$ denote the axial, transverse, axial shear moduli and axial Poisson's ratio of a laminate containing $n$ cracks per unit length. The values of these constants can be derived from the generalised plane strain model of stress transfer described above, including for cases when the cracks present are randomly rather than uniformly spaced. The values of the longitudinal, transverse and shear strains are obtained from (6) by differentiation leading to stress-strain relations of the form: 


$$
\begin{gathered}
\varepsilon=-\frac{\partial g}{\partial \sigma}=\frac{\sigma}{E_{A}(n)}-\frac{v_{A}(n)}{E_{A}(n)} \sigma_{t}+\alpha_{A}(n) \Delta T, \\
\varepsilon^{*}=-\frac{\partial g}{\partial \sigma_{t}}=-\frac{v_{A}(n)}{E_{A}(n)} \sigma+\frac{\sigma_{t}}{E_{T}(n)}+\alpha_{T}(n) \Delta T, \\
\gamma=-\frac{\partial g}{\partial \tau}=\frac{\tau}{\mu_{A}(n)},
\end{gathered}
$$

where $\gamma$ is the engineering strain

$$
\begin{aligned}
& \frac{\sigma_{o}}{E_{A}(n)}-\frac{v_{A}(n)}{E_{A}(n)} \sigma_{o}^{*}+\alpha_{A}(n) \Delta T=0, \\
& -\frac{v_{A}(n)}{E_{A}(n)} \sigma_{o}+\frac{\sigma_{o}^{*}}{E_{T}(n)}+\alpha_{T}(n) \Delta T=0 .
\end{aligned}
$$

If $\sigma \leq \sigma_{\mathrm{o}}$ and $\sigma_{\mathrm{t}} \leq \sigma_{\mathrm{o}}^{*}$ then the relations (6-11) are valid only if the parameter $\mathrm{n}$ is set to zero indicating that values of the damage-free thermoelastic constants must then be used.

If $\mathbf{p}$ cracks per unit length have just formed in the $90^{\circ}$ ply of the laminate that previously contained $\mathbf{n}$ cracks per unit length then $\Delta \Gamma=2 \gamma_{\mathrm{t}} \mathrm{Ap}$, where $2 \gamma_{\mathrm{t}}$ is the fracture energy for transverse cracking and $\mathrm{A}$ is the area of cracking associated with just one crack in the $90^{\circ}$ ply of the laminate. It follows from (6) that the corresponding change of Gibbs free energy for volume $\mathrm{V}$ of the laminate is given by:

$$
\begin{aligned}
\Delta G= & -\frac{V}{2}\left[\left(\sigma-\sigma_{0}\right)^{2}\left(\frac{1}{E_{A}(n+p)}-\frac{1}{E_{A}(n)}\right)+\left(\sigma_{t}-\sigma_{o}^{*}\right)^{2}\left(\frac{1}{E_{T}(n+p)}-\frac{1}{E_{T}(n)}\right)\right. \\
& \left.-2\left(\sigma-\sigma_{0}\right)\left(\sigma_{t}-\sigma_{0}^{*}\right)\left(\frac{v_{A}(n+p)}{E_{A}(n+p)}-\frac{v_{A}(n)}{E_{A}(n)}\right)+\tau^{2}\left(\frac{1}{\mu_{A}(n+p)}-\frac{1}{\mu_{A}(n)}\right)\right] .
\end{aligned}
$$

It then follows from the energy balance equation (5) that

$$
\begin{aligned}
2 \Gamma p= & \frac{1}{2}\left(\sigma-\sigma_{o}\right)^{2}\left(\frac{1}{E_{A}(n+p)}-\frac{1}{E_{A}(n)}\right)+\frac{1}{2}\left(\sigma_{t}-\sigma_{o}^{*}\right)^{2}\left(\frac{1}{E_{T}(n+p)}-\frac{1}{E_{T}(n)}\right) \\
& -\left(\sigma-\sigma_{o}\right)\left(\sigma_{t}-\sigma_{o}^{*}\right)\left(\frac{v_{A}(n+p)}{E_{A}(n+p)}-\frac{v_{A}(n)}{E_{A}(n)}\right)+\frac{1}{2} \tau^{2}\left(\frac{1}{\mu_{A}(n+p)}-\frac{1}{\mu_{A}(n)}\right),
\end{aligned}
$$

where $\Gamma=\gamma_{1} \mathrm{a} / \mathrm{h}$ is the effective fracture energy; $\mathrm{h}$ denoting the laminate half-thickness. In passing, it is worth commenting that the result (13) involves only macroscopic parameters, namely the thermoelastic 
constants of a cracked laminate and the stresses $\sigma_{0}$ and $\sigma_{0}^{*}$ required to just close any cracks that are present in the $0^{\circ}$ and $90^{\circ}$ plies. The result (13) can be used to investigate simultaneous cracking by setting $\mathbf{n}=0$, and progressive cracking by setting $\mathrm{n}=\mathrm{p}$ for the case of regular cracking leading to uniform crack densities. Random cracking can be predicted only if some statistical variability is introduced. In [20] such variability is introduced by assuming that the fracture energy $\Gamma$ is statistically distributed, and that cracks form one at a time. For this case (13) can again be used on setting $\mathbf{p}=1$.

For the case of biaxial loading, and uniaxial loading in axial and transverse directions, the relation (13) has been used to predict $[18,20]$ simultaneous and progressive cracking in 0-90-0 laminates for the cases of regular (always having a uniform crack density) and random crack formation. It is found that when predicting the initiation of transverse cracking the transverse cracking stress has a minimum value at a specific value of crack density (assumed uniform) that is independent of the length of laminate considered provided it is long enough. Clearly, the minimum value of the cracking stress at the preferred crack density is lower than would be calculated if only one crack was assumed to form in a laminate of any length. It is worth noting that shear-lag stress transfer models do not lead to this behaviour.

The above energy balance approach to predicting the criterion for crack formation under complex loading conditions is not able to provide expressions for the thermoelastic constants as a function of the number of transverse cracks per unit length. This must be provided by the generalised plane strain model of stress transfer [16]. However, the characteristics of the model are such that both stress and displacement distributions can be found and used to derive directly the fundamental relations (7-12). This derivation is based on the identification of effective axial and transverse strains with axial and transverse displacements at the edges of the laminate. Furthermore, the complete solution model can be used to calculate the Gibbs free energy of a cracked laminate directly by integrating the Gibbs free energy densities over the regions of the cracked laminate occupied by the $0^{\circ}$ and $90^{\circ}$ plies. The relation (12) results from such calculations [20] and consistency with the energetic approach described above is demonstrated. The ability of a micromechanical model to demonstrate energetic consistency can be used as a measure of its quality, and to indicate those situations where micromechanical models of stress transfer are needed only to derive expressions for the thermoelastic constants as a function of crack density.

\section{CONCLUSIONS}

1. Because of the many approximations that have to made, and because higher quality stress transfer models are now becoming available, shear-lag theories of stress transfer are not recommended for use as a basis for predicting crack initiation and growth in composite materials.

2. By employing complete solution models of stress transfer for unidirectional and 0-90-0 laminates, together with energy balance principles, relations that predict simultaneous and progressive growth of cracking can be derived that can be expressed in terms of macroscopic parameters, namely, the thermoelastic constants of cracked composites. The relatively simple form of such relations will lead to the development of new design methods for dealing with the effects of damage on the properties of composite materials. Furthermore, micromechanics is needed only to predict the dependence of the thermoelastic constants on crack density.

3. Stress-strain behaviour, and the dependence of stress, strain and the thermoelastic constants on crack density can be determined and used to assess the effects of damage on composite materials for cases of regular and random crack formation. 


\section{ACKNOWLEDGEMENT}

The research in this paper was carried out as part of the 'Materials Measurement Programme', a programme of underpinning research financed by the UK Department of Trade and Industry.

\section{REFERENCES}

[1] COX H.L., Br. J. Appl. Phys. 3 (1952) 72.

[2] NAYFEH A.H., Fib. Sci. Tech. 10 (1977) 195.

[3] NUISMER R.J. \& TAN S.C., J. Comp. Mater. 22 (1988) 306.

[4] McCARTNEY L.N., Proc. IUTAM Symposium, Springer-Verlag (1991) Blacksburg Va. ed. J.N Reddy \& K L Reifsnider, p.251.

[5] REISSNER E., J. Math. Phys. 29 (1950) 90.

[6] HASHIN Z., Mech. Mater. 4 (1985) 121.

[7] HASHIN Z., J. AppI. Mech. 54 (1987) 872.

[8] HASHIN Z., Comp. Sci. Tech. 31 (1988) 247.

[9] NAIRN J., Mech. Mater. 13 (1992) 131.

[10] PAGANO N.J., Proc. IUTAM Symposium, Springer-Verlag (1991) Blacksburg Va. ed. J.N Reddy \& K L Reifsnider, p.1.

[11] PAGANO N.J. \& BROWN H.W., Composites 24 (1993) 69.

[12] PAGANO N.J., Int. J. Solids Struct. 14 (1978) 385

[13] McCARTNEY L.N., Proc. Roy. Soc. Lond. A425 (1989) 215.

[14] McCARTNEY L.N., (1992) to be published.

[15] McCARTNEY L.N., (1993) to be published.

[16] McCARTNEY L.N., J. Mech. Phys. Solids 40 (1992) 27.

[17] PAGANO N.J., (1992) Private communication.

[18] McCARTNEY L.N., Composites 24 (1993) 84.

[19] AVESTON J., COOPER G.A. and KELLY A., In Conference on properties of fibre composites, National Physical Laboratory, IPS Science \& Technology, Guildford, Surrey (1971) p.15.

[20] McCARTNEY L.N., (1993) to be published. 